\title{
Monitoring and Productive Efficiency in Public and Private Firms *
}

\author{
Anke S. Kessler \\ University of Bonn \\ Christoph Lülfesmann ${ }^{\dagger}$ \\ and CEPR \\ University of Bonn
}

January 2001

\begin{abstract}
The paper compares productive efficiency in public and private firms. We study a principalagent model in which the firm's manager is privately informed about a cost parameter and exerts unobservable cost reducing effort, while the owner can conduct costly audits to obtain information about the firm's cost. Without auditing, managerial effort (and therefore production efficiency) is strictly higher under public governance with a benevolent government. If auditing is possible, however, a profit-maximizing private owner always audits at least as frequently as a public principal. For small auditing costs, we find that monitoring decisions, managerial effort and welfare under both governance structures coincide. Conversely, when audits becomes more expensive, the public (but not the private) owner refrains from monitoring, and the private firm may produce more cost efficiently.
\end{abstract}

Keywords: Production Efficiency, Privatization, Monitoring.

JEL-Classification: D23, L23, L33

*We wish to thank various conference participants of the 2000 Conference on Public Economic Theory in Warwick and Dieter Bös for helpful comments and discussions. Remaining errors are our own.

$\dagger$ Corresponding Author; Address: University of Bonn, Finanzwissenschaftliche Abteilung, Adenauerallee 24-42, 53113 Bonn, Germany, e-mail: clmann@wiwi.uni-bonn.de, phone: +49-228-737939, fax: $+49-228-739239$. 


\section{Introduction}

Does private ownership promote the internal efficiency of firms? One major reason for the privatization programs pursued throughout Europe in the past decades was the belief that private enterprises operate more efficient than their public counterparts. To some extent, empirical evidence lends support to this popular view. According to estimation results in Megginson et al. (1994) and Boubraki and Cosset (1998), for example, firm profitability (defined as net income divided by sales) increases from an average value of 8.6 percent before to 12.6 percent after privatization. Likewise, sales per employee rise on average by about 25 percent in the first three years after the change in governance structure occurred. ${ }^{1}$

The theoretical literature has offered some explanations for this phenomenon. Alchian and Demsetz (1972) and Vickers and Yarrow (1991) reason that due to the vague and frequently changing objectives of public relative to private owners, the efficient use of human resources and other inputs is monitored to a much lesser extent than in private firms. Similarly, Bös (1991) argues that the lack of monetary incentive schemes promotes managerial slack in public enterprises. These positions have an intuitive appeal. Yet, they attribute the relative internal efficiency advantages of private ownership solely to the unwillingness or inability of the government to exert appropriate control over the managers in a public enterprise. ${ }^{2}$

It is thus natural to ask whether public firms produce less cost-efficiently than private ones even if those deficiencies can be overcome. In the present paper, we neglect issues such as unclear objectives and inefficient contracting. Instead, we model the government as a fully rational actor who has access to the same information and contractual possibilities than a private entrepreneur. We maintain the presumption that both private and public enterprises face an agency problem associated with the separation of ownership and control. Specifically, our framework is based on a principal-agent relationship between the respective (public or private) owner of the firm on the one hand, and the firm's managers or workers on the other hand. The latter have private information on

\footnotetext{
${ }^{1}$ As some studies [e.g., Boardman and Vining (1989)] also find instances where productivity fell after privatization, however, the overall evidence is not fully conclusive. A survey on empirical work on this issue is provided by Megginson and Netter (1999).

${ }^{2}$ In addition, the above line of reasoning implies that one should also expect inefficiencies in privately owned but regulated firms. If regulatory policy is affected by the government's lack of control as well, however, privatization in non-competitive industries becomes less attractive.
} 
the firm's intrinsic productivity and can exert unobservable effort to reduce the total cost of production. In order to reduce this agency problem, the principal can give incentives through monetary compensation schemes. In addition, she can conduct audits. Private and public firms differ only with respect to the objectives of the respective principal: while the government as owner of a public firm maximizes welfare, ${ }^{3}$ the private owner is purely profit-oriented. Throughout the analysis, it is important to keep in mind that we do not (and cannot) address the issue of privatization versus nationalization directly, although some results naturally can be applied to this context as we argue below. ${ }^{4}$ Instead, we simply ask whether and under which circumstances the productive efficiency as measured by managerial effort in a private firm can be higher than in a publicly owned company.

Due to the difference in objectives, a social-welfare maximizing public owner is willing to pay higher incentive compensations than a profit-maximizing private owner: because the former also cares about the utility of her employees, the tension between productive efficiency and rent extraction is less pronounced in a publicly-held firm. In a situation where auditing is not possible, therefore, the government implements higher managerial effort than the private principal. $^{5}$ As we show, this conclusion no longer holds once the audits are taking into account. Precisely because leaving rents is more costly to a private owner, she always audits at least as frequently as a public owner. Whereas high managerial compensation is used as a 'carrot' to give incentives in public firms, more audits are conducted and used as a 'stick' in private firms. In particular, there are circumstances where auditing enables the private principal to induce higher costreducing effort than the government (who chooses not to audit at all) without granting an informational rent to the manager. Then, the private firm operates with strictly higher cost efficiency than a public enterprise. Finally, we identify situations where the outcomes under either governance structure coincide, namely, if auditing is relatively

\footnotetext{
${ }^{3}$ While this assumption is strong and even unrealistic, it allow us to focus on the question whether productive efficiency in a public firm is low even in a world where government officials are driven by idealistic motives, or are perfectly controlled by their constituency.

${ }^{4}$ Since we admit complete, fully contingent contracts, our model is not suited to analyze whether privatization can be strictly welfare improving. In particular, there exists an optimal privatization and regulation procedure which perfectly aligns the objectives of the private owner with those of the government [see also Sappington and Stiglitz (1987)]. Under the optimal scheme, privatization therefore does not affect the productive and allocative efficiency of the firm. In contrast, if contracts are incomplete private ownership may be strictly superior even if government is benevolent. See, for example, Schmidt (1996a,b), Hart et. al (1997), and Segal (1998).

${ }^{5}$ See the paper by de Fraja (1993) which is discussed in more detail below.
} 
inexpensive and therefore takes place under both ownership structures. Public and private owner then take the same decisions as long as the private firm is regulated on its output market, or if this output market is competitive so that external regulation is not necessary. ${ }^{6}$

A variety of contributions use agency settings with informational asymmetries to study internal efficiency under private and public (benevolent) governance. Most closely related to the present paper is de Fraja (1993) who provides the first comparison of internal efficiency under both ownership structures. The author analyzes an adverse-selection model similar to ours, but does not consider auditing as a means to reduce the prevailing incentive problem. He shows that a welfare maximizing government values a reduction in managerial rents less and productive efficiency more than a private owner. As a consequence, the public principal induces higher cost-reducing effort in equilibrium. A similar conclusion is drawn in Laffont and Tirole (1991) who argue that privately owned but regulated firms suffer from a common agency problem between shareholders and regulators which can cause managerial slack to be higher under privatization. While both papers find that internal efficiency is lower in private as compared to public firms, a result that corresponds to ours is obtained independently in Corneo and Rob (2000). The authors consider a multi-task model in which workers allocate there time between individual production and 'socializing'. The former activity is costly and the latter both yields utility for the workers and positively affects output. Because a benevolent public owner is willing to tolerate a higher amount of socializing than a private owner, productivity may (but need not) be higher under private governance.

The present paper is also related to some work on incomplete-contracting, which shows that privatization can be the optimal mode of governance. Schmidt (1996a, 1996b) introduces a connection between ownership and information structure. Privatization creates an informational wedge which can be beneficial even if the public principal is fully benevolent: it enables the government to commit ex post to a harder budget constraint, thereby improving a manager's incentives to invest in (non-contractible) cost reduction

\footnotetext{
${ }^{6}$ In this case, privatization does not require a sophisticated contract to ensure the optimal managerial effort. A simple sales scheme in the form of a lump-sum payment from the new owner to the government and - if the firm does not operate in a competitive environment - an appropriate regulatory component will produce the second-best efficient result. This result shows that optimally designed privatization schemes need not always be complex, i.e., there are circumstances where the government does not have to exert (possibly indirect) control on the firm's internal arrangements such as managerial incentive schemes.
} 
ex ante. Hart, Shleifer and Vishny (1997) demonstrate in a property rights model that a private owner expends a larger effort than a public manager because he becomes residual claimant for realized cost savings in a bargaining game vis-a-vis the government. A similar effect emerges in Schmitz (2000) who, however, shows that partial privatization (government and management jointly own the firm) may dominate full privatization.

The paper proceeds as follows. Section 2 presents the model, and derives optimal auditing and contracting decisions for both ownership structures under consideration. Section 3 compares both regimes and relates productive efficiency to ownership. Section 4 concludes.

\section{The Model}

Consider the following simple version of a principal-agent adverse selection model with managerial effort and monitoring. The principal owns a productive unit and hires a manager or worker $(\mathrm{M})$ to realize an indivisible project at a $\operatorname{cost} C=\theta-e$, where $\theta$ is a random parameter that represents the firm's intrinsic productivity (or, equivalently, the managers ability) and $e \in \mathbb{R}_{0}^{+}$is managerial effort. For simplicity, we assume that $\theta$ can take on only two values $\bar{\theta}>\underline{\theta}$ with $q \in(0,1)$ denoting the commonly known ex ante probability that the firm's costs are low. When exerting $e$, the manager reduces the firm's costs of production (e.g., organizational slack) but incurs a disutility which is represented in monetary terms by an increasing and strictly convex function $\psi(e)$ which satisfies $\psi(0)=\psi^{\prime}(0)=0$ and $\psi^{\prime \prime \prime}(\cdot) \geq 0 .{ }^{7}$ While the firm's cost $C$ is publicly observable, both $\theta$ and $e$ are private information of the manager. The principal can, however, collect information on the firm's internal efficiency through auditing. Following Kofman and Lawarrée (1993), auditing generates a verifiable signal $s \in\{\bar{s}, \underline{s}\}$ which is (imperfectly) correlated with $\theta$. The probability that the signal is correct is independent of the realization of $\theta$ and denoted by $\gamma$, i.e. $\operatorname{Prob}\{s=\bar{s} \mid \theta=\bar{\theta}\}=\operatorname{Prob}\{s=\underline{s} \mid \theta=\underline{\theta}\}=$ $\gamma$. With probability $1-\gamma$, the observation is incorrect. Without loss of generality, let $\gamma>\frac{1}{2} \cdot{ }^{8}$

\footnotetext{
${ }^{7}$ The assumption on the third derivative of $\psi(\cdot)$ ensures that the principal's problem is well behaved (see, e.g., Laffont and Tirole (1986).

${ }^{8}$ As will become clear below, none of the subsequent results qualitatively depends on this particular specification of the monitoring technology. For a model where the principal can observe the manager's effort rather than $\theta$, see, e.g., Laffont and Tirole (1992) and Kessler (2000).
} 
The principal may be a welfare maximizing government $(\mathrm{G})$ or a private owner $(\mathrm{P})$. Her respective benefit from production is $S_{j}>0, j \in\{G, P\}$. Although we allow $S_{G}$ and $S_{P}$ to differ, we will throughout the paper maintain the assumption that both valuations are sufficiently large so that the project should always be realized, irrespective of the firm's cost and ownership or informational structure. ${ }^{9}$ The principal reimburses the firm's costs $C$ (an accounting convention) and offers incentive contracts to the manager that can condition his remuneration $w$ on commonly observable variables such as $C$ and - in case of an audit $-s$. The manager's utility is

$$
U=w-\psi(e)
$$

if he works for the principal and equal to his reservation utility $U_{r}$ otherwise. The government is utilitarian but faces a shadow cost of public funds $\lambda>0$. Using (1), its objective function can be written as ${ }^{10}$

$$
\begin{aligned}
u_{G} & =S_{G}-(1+\lambda)(C+w)+U \\
& =S_{G}-(1+\lambda)\left[\theta-e+\psi(e)+\frac{\lambda}{1+\lambda} U\right]=S_{G}-(1+\lambda) \mathcal{C}_{G},
\end{aligned}
$$

where $\mathcal{C}_{G}=\theta-e+\psi(e)+\frac{\lambda}{1+\lambda} U$ are the total costs of production, including managerial compensation. In contrast, the private principal maximizes profits,

$$
u_{P}=S_{P}-C-w=S_{P}-[\theta-e+\psi(e)+U]=S_{P}-\mathcal{C}_{P}
$$

with $\mathcal{C}_{P}=\theta-e+\psi(e)+U$. The game proceeds as follows. In stage 0 , the principal decides whether to invest $I \geq 0$ in the auditing technology. One should think of this investment as fixed expenses that enable her to subsequently audit the firm such as setting up an accounting division or other institutional structures facilitating internal control. In stage 1, the principal offers an employment contract to the manager, which specifies a remuneration $w$ and the probability $p$ of a subsequent audit. If the manager accepts, he subsequently exerts effort and carries out production in stage 2. Having observed realized cost $C$, the principal may conduct an audit, which imposes additional

\footnotetext{
${ }^{9}$ Equivalently, the private firm may be regulated on its output market such that the private owner's valuation of output coincides with that of the government [see also footnote 11 below].

${ }^{10}$ See Laffont and Tirole $(1986,1992)$. We could could alternatively follow Baron and Myerson (1982) who assume that the social welfare maximizing government attaches a relative weight $\beta<1$ on the managerial surplus relative to consumer surplus. Note that both formulations are formally equivalent if one sets $\beta=\frac{\lambda}{1+\lambda}$.
} 
costs $c>0$ and generates the commonly observed signal $s$. Finally, payoffs are realized in stage 4 .

Before proceeding, let us briefly consider the benchmark case where $\theta$ is publicly observable. Under symmetric information on $\theta$, the principal maximizes her utility (2) or (3), subject to the manager's participation constraint $U \geq U_{r}$. Thus, the first-best contract $\left(e^{*}, U^{*}\right)$ is characterized by $\psi^{\prime}\left(e^{*}\right)=1$ and $w^{*}=\psi\left(e^{*}\right)+U_{r}$, irrespective of $\theta$ and the governance structure. This property of the first-best contract facilitates a comparison between the two ownership structures for the case where $\theta$ is private information of the manager. It is an immediate consequence of our assumption that the project is of fixed size and will be realized irrespective of ownership. ${ }^{11}$

We now turn to the more interesting case where $\theta$ is private information of the manager. We begin by solving for the second-best solution without auditing:

\section{Optimal Contracts without Auditing}

If the principal has at stage 0 decided not to invest into auditing, her problem is a standard adverse selection problem. Let $(\underline{w}, \underline{C})$ and $(\bar{w}, \bar{C})$ be the contracts for a manager who claims that the cost parameter is $\underline{\theta}$ and $\bar{\theta}$, respectively. Using (1) and setting $e=\theta-C$ the manager's participation and incentive compatibility constraints can be written as

$$
\begin{array}{lll}
\bar{U} \geq U_{r} & (\overline{P C}) & \underline{U} \geq U_{r} \\
\bar{U} \geq \underline{U}+\psi(\underline{e})-\psi(\underline{e}+\Delta \theta) & (\overline{I C}) & \underline{U} \geq \bar{U}+\psi(\bar{e})-\psi(\bar{e}-\Delta \theta) .
\end{array}
$$

where $\Delta \theta \equiv \bar{\theta}-\underline{\theta}>0$. Subject to the (PC) and (IC) constraints, the principal maximizes her expected payoff.Since the project should always be realized, this is equivalent to minimizing expected total production cost, which can be obtained from (3) and (2), respectively. It is now straightforward to show that the optimal contract with no audits $(n a)$ is characterized as follows [see also Laffont and Tirole (1993)]. While either principal implements the first-best effort in the low-cost state $\left(\underline{e}_{j}^{n a}=e^{*}, j=G, P\right)$, the induced

\footnotetext{
${ }^{11}$ If production $x$ is variable, the relative magnitudes of the respective (marginal) benefits from production, $d S_{G} / d x$ and $d S_{P} / d x$, must be taken into account. For example, suppose that the firm has market power and its output is not regulated under private governance. Then, the government's marginal benefit from production (the sum of consumer and producer surplus) exceeds that of a private owner. In such a situation, production will be higher under public ownership, and so will be the marginal value of cost reduction. Hence, a first best contract would specify $e_{G}^{*}>e_{P}^{*}$, making the analysis of the cost-efficiency consequences of governance in the presence of informational asymmetries less transparent.
} 
effort level if costs are high is determined by the first order conditions ${ }^{12}$

$$
\begin{aligned}
& 1=\psi^{\prime}\left(\bar{e}_{G}^{n a}\right)+\frac{\lambda}{1+\lambda} \frac{q}{1-q}\left[\psi^{\prime}\left(\bar{e}_{G}^{n a}\right)-\psi^{\prime}\left(\bar{e}_{G}^{n a}-\Delta \theta\right)\right] \\
& 1=\psi^{\prime}\left(\bar{e}_{P}^{n a}\right)+\frac{q}{1-q}\left[\psi^{\prime}\left(\bar{e}_{P}^{n a}\right)-\psi^{\prime}\left(\bar{e}_{P}^{n a}-\Delta \theta\right)\right] .
\end{aligned}
$$

Closer inspection of (4) and (5), reveals that $\bar{e}_{P}^{n a}<\bar{e}_{G}^{n a}$ for all finite values of $\lambda$, i.e., public ownership results in a higher degree of internal efficiency in this state. This finding essentially replicates the argument of de Fraja (1993). Intuitively, the trade-off between efficiency and rent extraction is less pronounced for the public than for the private owner because the government also cares about the utility of the management or workforce in the firm. ${ }^{13}$ As a consequence, the public principal leaves higher rents to the firm's manager than a private owner in the low-cost state. To see this, note that from $(\overline{P C})$ and $(\underline{I C})$,

$$
\underline{U}_{j}^{n a}=U_{r}+\psi\left(\bar{e}_{j}^{n a}\right)-\psi\left(\bar{e}_{j}^{n a}-\Delta \theta\right)>U_{r} \quad \text { and } \quad \bar{U}_{j}^{n a}=U_{r} \quad j \in\{G, P\} .
$$

As the informational rent $\phi\left(\bar{e}_{j}^{n a}\right) \equiv \psi\left(\bar{e}_{j}^{n a}\right)-\psi\left(\bar{e}_{j}^{n a}-\Delta \theta\right)>0$ strictly increases in $e$, $\bar{e}_{G}^{n a}>\bar{e}_{P}^{n a}$ implies $\phi_{G}^{n a}>\phi_{P}^{n a}$.

\section{Optimal Contracts with Auditing}

Now suppose the principal has invested into auditing in stage 0 . This enables her to observe a signal $s$ on $\theta$ which is informative and - on average - correct due to $\gamma>\frac{1}{2}$. To derive the optimal contract, note first that if the manager reports low costs $\underline{\theta}$, there is no need for an audit to take place. If the manager announces high costs $\bar{\theta}$, however, he may have misreported the firm's true productivity and it may now be useful for the principal to request an audit. Recall that $p \in[0,1]$ denotes the contractually specified probability with which auditing occurs. Again, we assume that the principal can commit to her initial contract offer so that the revelation principle continues to apply. ${ }^{14}$ If the audit

\footnotetext{
${ }^{12}$ The $(\underline{I C})$ constraint implicitly requires $\bar{e}>\Delta \theta$. One therefore has to assume $\psi^{\prime}(\Delta \theta)<1-q$.

${ }^{13}$ De Fraja (1993) considers a more general framework with variable production. In his model, the welfare-maximizing government has a higher marginal surplus from additional output (assuming the private firm is not regulated). Therefore, a second - and reinforcing - effect applies since the government's benefit from an increase in $e$ exceeds that of a private principal even if $\lambda \rightarrow \infty$.

${ }^{14}$ If the principal cannot commit to her auditing strategy, the revelation principle no longer holds and mixed strategy equilibria may emerge which involve shirking (non-compliance) on the part of the agent [see, e.g., Cowell (1984) and Khalil (1997)]. While the basic trade-off between rent extraction and efficiency on which our results depend still applies, the analysis is rather complex and beyond the scope of the present paper.
} 
yields a low-cost signal $\underline{s}$, indicating that the manager has concealed the firm's true costs, he can be punished. We assume that in this case, the principal collects an exogenously given fine $F>0 .{ }^{15}$ If we continue to denote the manager's gross remuneration by $w$, an honest manager's utility in the high-cost state is now $\bar{U}=\bar{w}-\psi(\bar{e})-p(1-\gamma) F$. In the low-cost state, he receives $\underline{w}-\psi(\underline{e})=\underline{U}$ if he reported cost truthfully and $\bar{w}-\psi(\bar{e}-\Delta \theta)-p \gamma F$ otherwise. The low-cost manager's $(I C)$-constraint thus becomes $\underline{w}-\psi(\underline{e}) \geq \bar{w}-\psi(\bar{e}-\Delta \theta)-p \gamma F$, or

$$
\underline{U} \geq \bar{U}+\psi(\bar{e})-\psi(\bar{e}-\Delta \theta)-p(2 \gamma-1) F .
$$

It is useful to define two critical values for the principal's (marginal) cost $c$ of audits,

$$
c_{G} \equiv \frac{q}{1-q} \frac{\lambda}{1+\lambda}(2 \gamma-1) F \quad \text { and } \quad c_{P} \equiv \frac{q}{1-q}(2 \gamma-1) F
$$

with $c_{P}>c_{G}>0$. The proposition below summarizes the formal analysis of the optimal contract with auditing that can be found in the Appendix.

Proposition 1. Suppose that the principal incurs I at stage 0 so that subsequent audits become feasible. Under either ownership structure $j \in\{G, P\}$, managerial effort is efficient in the low cost state, $\underline{e}_{j}^{a}=e^{*}$, and the manager earns no rents in the high cost state, $\bar{U}_{j}^{a}=U_{r}$. If $c>c_{j}$, the principal $j$ refrains from auditing $\left(p_{j}=0\right)$ and the optimal contract is identical to the second-best contract without auditing. For $c \leq c_{j}$, auditing occurs with positive probability $\left(p_{j}>0\right)$ and we have

a) $\bar{e}_{j}^{a}=\bar{e}_{j}^{n a}$ and $\underline{U}_{j}^{a}<\underline{U}_{j}^{n a}$ for values $(2 \gamma-1) F \leq \phi_{j}^{n a}$

b) $e^{*}>\bar{e}_{j}^{a}>\bar{e}_{j}^{n a}$ and $\underline{U}_{j}^{a}=U_{r}$ for values $(2 \gamma-1) F>\phi_{j}^{n a}$.

Moreover, public and private contracting are characterized by $p_{P} \geq p_{G}$ and $\bar{e}_{P}^{a} \leq \bar{e}_{G}^{a}$ with

$$
\bar{e}_{G}^{a}>\bar{e}_{G}^{n a} \Rightarrow \quad p_{G}=p_{P}>0 \quad \text { and } \quad \bar{e}_{G}^{a}=\bar{e}_{P}^{a} .
$$

Not surprisingly, auditing allows the principal to decrease informational rents. Also, if the rent is already extracted at $\bar{e}_{j}^{n a}$ for a given fine $F$ and signal precision $\gamma$, the

\footnotetext{
${ }^{15}$ While the principal may be able to freely chose the extent of the penalty, we know from the maximum deterrence principle [Baron and Besanko (1984)] that she will always choose the highest possible fine. Hence, $F$ can be interpreted as the maximum amount the agent is (legally) liable for. Alternatively, one could interpret the agent's limited liability as a wealth constraint as, e.g., in Laffont and Tirole (1992). Penalties are then transfer dependent: the higher the agent's remuneration, the more can he be held liable. This additional effect adds complexity without qualitatively changing our results.
} 
principal optimally implements higher managerial effort in the high-cost state, thereby improving productive efficiency [part b)]. Otherwise, managerial effort is unaffected by the possibility of audits under the optimal contract [part a)]. ${ }^{16}$ The last part of Proposition 1 compares private and public optimal contracting if both principals have invested into auditing and yields two observations. First, the private principal always audits at least as frequently as the public principal. Second, if public monitoring strictly improves cost efficiency $\left(\bar{e}_{G}^{a}>\bar{e}_{G}^{n a}\right)$ both types of principal audit equally often and the internal efficiency does not differ across governance structures. The intuition for these results is as follows: for $U_{j}^{a}=U_{r}$, a further reduction in $U$ is impossible and both principals $j$ attach the same value to an increase in productive efficiency [see (2) and (3)]. Because the marginal benefit from auditing and from raising $\bar{e}_{j}$ is independent of ownership $j$, optimal contracts coincide. For $\underline{U}_{j}^{a}>U_{r}$, on the other hand, a private owner's valuation for a marginal reduction in the manager's informational rent exceeds that of the government and thus $p_{P} \geq p_{G}$. Yet, managerial effort $\bar{e}_{j}$ is lower in private than in public contracting since it is unaffected by the auditing decision and equal to the effort induced without audits.

To summarize, the private principal's marginal benefit from auditing always weakly exceeds that of her public counterpart. ${ }^{17}$ Loosely speaking, a private owner prefers to use auditing and the associated fine as a 'stick' to induce the manager to exert costreducing effort. The public principal, in contrast, prefers to give incentives with a 'carrot' even if the stick is available. She audits less and leaves higher rents to the manager that induce him to work hard. Furthermore, if public auditing occurs and improves productive efficiency, both ownership structures are equally cost-effective.

\section{Optimal Monitoring and Productive Efficiency}

We are now in a position to compare internal efficiency under public and private ownership by analyzing in which situations either principal will actually invest into the auditing technology in stage 0 . It should be emphasized that the timing of this decision is irrelevant for the results that follow. In particular, one could also view the investment $I$ as a

\footnotetext{
${ }^{16}$ See also Kofmann and Lawarrée (1993) who discuss the properties of the optimal contract for a private principal in more detail.

${ }^{17}$ Hence, the conclusion that $p_{P} \geq p_{G}$ is more general than suggested in the proposition. It holds for any specification of the marginal auditing cost $c(p)$, not only for $c^{\prime}(p)=0$ as we have assumed here.
} 
fixed cost associated with audits, which the principal only has to incur if those actually take place in stage 3. For ease of exposition, however, we will continue to interpret $I$ as an investment that must be made ex ante, before contractual arrangements are made. From Proposition 1, it should be clear that a principal $j$ will not expend $I$ when the marginal costs of monitoring exceed $c_{j}$, since in this case $p_{j}=0$ is sequentially optimal. More generally, because the principal $j$ is willing to invest in stage 0 if and only if her net benefit from conducting audits exceeds the cost $I$, the corresponding necessary and sufficient condition can be written as

$$
\mathcal{C}_{j}^{n a}-\mathcal{C}_{j}^{a} \geq I, \quad j=G, P
$$

where $\mathcal{C}_{j}^{a}$ and $\mathcal{C}_{j}^{n a}$ are the total expected production cost for the principal $j$ under the optimal contract with and without audits, respectively. ${ }^{18}$

Lemma 1. For any $\gamma \neq \frac{1}{2}$ and finite values of $\lambda$ and $F$, we have

$$
\mathcal{C}_{G}^{n a}-\mathcal{C}_{G}^{a} \equiv I_{G}<I_{P} \equiv \mathcal{C}_{P}^{n a}-\mathcal{C}_{P}^{a}
$$

The lemma establishes that the total benefit from auditing is strictly lower for the public than for the private principal, irrespective of the government's cost of raising public funds, the accuracy of audits, or the fine that can be imposed for shirking. ${ }^{19}$

To relate internal efficiency and ownership structures, recall that public and private principal always induce an efficient level of effort in the low-cost state. Hence, we can confine attention to the determination of cost efficiency in the high-cost state as measured by $\bar{e}_{j}$. Furthermore, since we already know that $\bar{e}_{G}^{n a}>\bar{e}_{P}^{n a}$, we will for expositional brevity ignore the case where $I$ is prohibitively high so that neither principal audits.

\footnotetext{
${ }^{18}$ Since the public principal takes her shadow cost of raising funds into account when evaluating the costs of auditing, we must have

$$
(1+\lambda)\left(\mathcal{C}_{G}^{n a}-\mathcal{C}_{G}^{a}\right) \geq(1+\lambda) I \quad \Leftrightarrow \quad \mathcal{C}_{G}^{n a}-\mathcal{C}_{G}^{a} \geq I
$$

for the investment to be optimal under public contracting.

${ }^{19}$ See the Appendix for a proof. Observe that Lemma 1 holds even though the informational rent that the government could maximally save through audits, $\phi_{G}^{n a}$, weighted by $\frac{\lambda}{1+\lambda}$ (that is, as valued by a private owner) may well exceed the corresponding maximal rent savings in the private firm, $\phi_{P}^{n a}$. The intuition is as follows. Suppose first that both principals monitor only to reduce the manager's rent. The thus achieved marginal rent reduction is $(2 \gamma-1) F$ which is clearly valued more by the private owner. Second, suppose private monitoring improves productive efficiency so that $U_{P}^{a}=U_{r}$. Then, the private principal always enjoys a higher increase in efficiency from auditing than the government (because the inefficiency is more pronounced under private ownership without monitoring) which overcompensates the effect that the private total benefit from rent reduction may be lower.
} 
Using Lemma 1, we therefore assume that $I \leq I_{P}$ in what follows. There are two cases to distinguish: suppose first that in equilibrium both public and private principal invest into the auditing technology, which is the case if $I \leq I_{G}$. Obviously, this can only be optimal if they both plan to subsequently conduct audits with positive probability. Hence, $I \leq I_{G}$ implies $c \leq c_{G}<c_{P}$ and therefore, $p_{P} \geq p_{G}>0$. Proposition $\left.1 \mathrm{~b}\right)$ then tells us that $\bar{e}_{G}=\bar{e}_{P}$ if public auditing improves internal efficiency, i.e., if $(2 \gamma-1) F>\phi_{G}^{n a}$. Otherwise, we have $\bar{e}_{G}>\bar{e}_{P}$. Since $\phi_{G}^{n a}$ is a monotonically decreasing function of $\lambda$, there exists a critical value $\tilde{\lambda} \geq 0$ such that $\bar{e}_{G}=\bar{e}_{P}$ if $\lambda>\tilde{\lambda}$ where $\tilde{\lambda}$ is implicitly defined by

$$
\phi\left(e_{G}^{n a}\right)=(2 \gamma-1) F
$$

with $e_{G}^{n a}$ given by (4) evaluated at $\lambda=\tilde{\lambda}$. Second, suppose $I \in\left(I_{G}, I_{P}\right]$ so that only the private principal (optimally) invests into auditing at all. This interval is nonempty for all conceivable parameter values of the model (Lemma 1) and the contractual arrangements then specify $\bar{e}_{G}=\bar{e}_{G}^{n a}$ and $\bar{e}_{P}=\bar{e}_{P}^{a}$ in equilibrium. Now, recall from Proposition 1 that $\bar{e}_{P}^{a}=\bar{e}_{G}^{a}>\bar{e}_{G}^{n a}$ if and only if $(2 \gamma-1) F>\phi_{G}^{n a}$. From our previous argument, this condition is equivalent to $\lambda>\tilde{\lambda}$ as defined above.

The proposition below compares productive efficiency under private and public ownership. It is a straightforward implication of Proposition 1, Lemma 1, and the preceding discussion:

Proposition 2. For $\lambda \leq \tilde{\lambda}$, either only the private principal invests in monitoring or public audits do not improve productive efficiency. In both cases, $e^{*}=\underline{e}_{G}=\underline{e}_{P}$ and $e^{*}>\bar{e}_{G} \geq \bar{e}_{P}$ with strict inequality if $\lambda<\tilde{\lambda}$. For any $\lambda \geq \tilde{\lambda}$, however, there exists a nonempty interval of fixed auditing costs $\left[I_{G}, I_{P}\right]$ such that under the optimal auditing and contracting decisions,

a) $I \leq I_{G}$ implies that both principals $j \in\{G, P\}$ invest into auditing, choose identical positive auditing probabilities $p_{j}$ and implement identical effort levels $\underline{e}_{j}=e^{*}$ and $\bar{e}_{j}>e_{j}^{n a}$.

b) $I_{G}<I \leq I_{P}$ implies that only the private principal monitors and internal efficiency is higher in the private than in the public firm,

$$
\underline{e}_{P}=\underline{e}_{G}=e^{*} \quad \text { and } \quad \bar{e}_{P}>\bar{e}_{G} .
$$

The first part of the proposition identifies situations where the productive efficiency of the private firm falls short of the internal efficiency in a publicly owned enterprise. 
Provided the shadow costs of public funds are sufficiently low $[\lambda<\tilde{\lambda}]$, cost reducing effort in a public firm strictly exceeds that in a private firm in the high-cost state even if monitoring possibilities are available. The second part a) establishes conditions where productive efficiency and social welfare are unaffected by the governance structure. This result demonstrates that there are situations in which welfare measures under either governance structure coincide. Provided that the firm's output market need not be regulated, optimal privatization arrangements can then take the form of a simple sales contract: the government can extract expected profits from a private entrepreneur by selling the firm for a predetermined price. Yet, even if output regulation is needed, it is not necessary for the government to intervene into the contractual arrangements between the private owner and her managers or any outside contractors. ${ }^{20}$

Finally, our main result on ownership and productive efficiency is stated in part b). Productive efficiency, measured by managerial effort in cost reduction, may be strictly higher in the private firm than in the public firm. It is important to note that this result does not contradict the argument that the public principal faces a weaker tension between rent extraction motives and productive efficiency than a profit-maximizing owner (although it qualifies the conclusion that the government therefore has a tendency to induce higher cost-reducing effort in equilibrium). Rather, precisely because of the public principal's lower valuation of informational rents, her incentives to monitor the manager in order to overcome the informational wedge between them fall short of those of a private profit-maximizing owner. If one takes the possibility of monitoring audits into account, therefore, there are situations in which only private owners rationally conduct audits and induce an equilibrium effort which is strictly higher than in a public firm.

The figure below illustrates these results. ${ }^{21}$ In the first region $\mathrm{A}$, for values of $\lambda<\tilde{\lambda}$, internal efficiency is higher in the publicly than in the privately owned firm, irrespective of whether or not audits occur with positive probability. Second, auditing decision, welfare, and equilibrium effort coincide in region B where $I \leq I_{G}$ and $\lambda \geq \tilde{\lambda}$. Note that global

\footnotetext{
${ }^{20}$ It is instructive to compare this finding with the statement of the "Fundamental privatization theorem" of Sappington and Stiglitz (1987). The theorem asserts that governance structure has no impact on efficiency, provided that the optimal contractual arrangement adjusts the incentives of the profit-maximizing owner to pursuing welfare objectives. Here, this arrangement would at most embody a regulatory component and there would be no need to interfere with the firm's internal affairs.

${ }^{21}$ The figure assumes that audits are a priori not prohibitively costly for either type of principal. It is drawn for the case where public auditing - if it is optimal - improves productive efficiency, i.e. $I \leq I_{G}$ already implies $\lambda>\tilde{\lambda}$.
} 
public expected benefit from audits, $I_{G}$, is monotonically increasing in the government's cost of public funds and approaches $I_{P}$ as $\lambda \rightarrow \infty$ so that the set of parameter values of $I$ for which ownership structure does not matter expands as $\lambda$ grows. Finally, for $I \in\left(I_{G}, I_{P}\right]$ the public principal strictly prefers not to audit, while auditing is still optimal for the private principal. If, in addition, $\lambda>\tilde{\lambda}$, we have $\bar{e}_{G}<\bar{e}_{P}$ as in region C. Hence, for intermediate values of $\lambda$ and $I$, private contracting with its associated audits implies strictly higher equilibrium effort than public contracting.

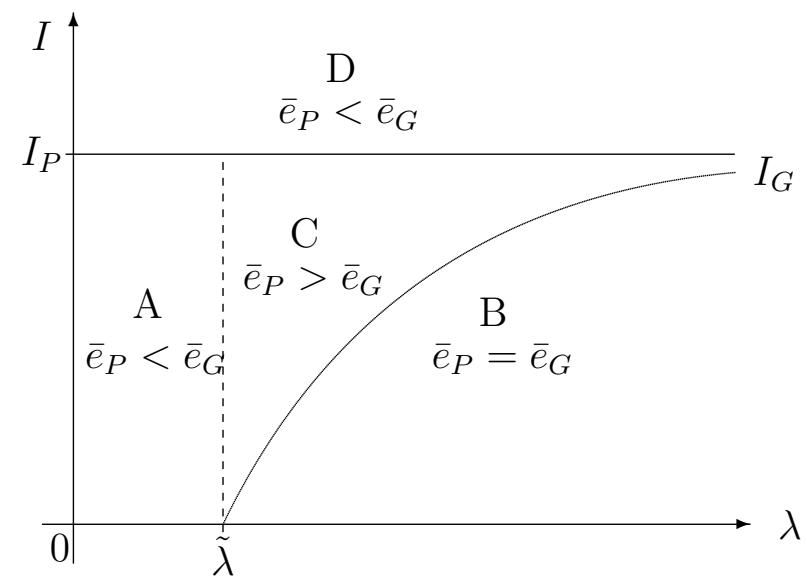

Ownership and Productive Efficiency

\section{Conclusion}

This paper has compared productive efficiency in public and privately owned enterprises. We have modelled the relationship between the respective (public or private) owner of the firm on the one hand, and the firm's managers or workers on the other hand, as a principal-agent relationship where the latter have private information on intrinsic costs and can exert unobservable cost-reducing effort. Allowing for the possibility of audits as a control instrument, three conclusions emerge from the analysis. First, a private profit-maximizing owner always conducts audits at least as often as a welfare-maximizing government. Hence, the model predicts different internal organization structures under either regime; auditing devices should be less frequently used in public as compared to private firms. Second, whenever public auditing strictly increases managerial effort in cost reduction, the internal efficiency of public and private firms coincides. Finally, we demonstrate that there are situations in which managerial effort is strictly higher in a 
private firm. Hence, productive efficiency may improve if a firm is privatized, which is well in line with empirical evidence as reported in various studies. Importantly, this result holds although the public principal is modelled as a (welfare-maximizing) rational actor who takes optimal auditing and contracting decisions.

Clearly, the government in practice is neither unitary, nor welfare-maximizing or even fully rational. It is composed of many actors with often conflicting interests, subject to pressure from interest groups, and suffers from inefficiencies in its administration and decision making process. In this regard, the model can only serve as an example that highlights the differences in governance structures which arise when the public principal does not pursue a pure profit objective. In other respects, however, the results obtained are quite general. In particular, they do not depend on the specific simple production and auditing technologies that were chosen to analyze the economic forces at work. What is important for the conclusions is that a) the government cares more about the utility of its subordinates than the private owner and b) the relationship between owner and employees is subject to incentive problems that give rise to rents. Since these conditions are likely to be satisfied in many situations, our work hopes to contribute to a better theoretical understanding of the virtues and vices of private and public sector performance. 


\section{References}

Alchian, A.A. and H. Demsetz (1972), "Production, Information Costs, and Economic Organization", American Economic Review, 62: 777 - 795.

Baron, D. and D. Besanko (1984), "Regulation, Asymmetric Information, and Auditing", RAND Journal of Economics, 15: 447 - 470.

Baron, D. and R. Myerson (1982), "Regulating a Monopolist with Unknown Costs", Econometrica, 50: 911-930.

Boardman, A. and A.D. Vining (1989), "Ownership and Performance in Competitive Environments: A Comparison of the Performance of Private, Mixed, and StateOwned Enterprises", Journal of Law and Economics, 32: 1-33.

Boubraki, N. and J.-C. Cosset (1998), "The Financial and Operating Performance of Newly-Privatized Firms: Evidence from Developing Countries", Journal of Finance, 53: $1081-1110$.

Bös, D. (1991), Privatization: A Theoretical Treatment, Oxford: Oxford University Press.

Corneo, G. and R. Rob (2000), "Working in Public and Private Firms," mimeo, University of Osnabrück.

Cowell, F. (1985), "The Economic Analysis of Tax Evasion," Bulletin of Economic Research, 37: 163-193.

De Fraja, G. (1993), "Productive Efficiency in Public and Private Firms", Journal of Public Economics, 50: 15 - 31.

Kessler, A.S. (2000), "On Monitoring and Collusion in Hierarchies", Journal of Economic Theory, 91: 280-291.

Hart, O., Shleifer, M. and R.W. Vishny (1997), "The Proper Scope of Government: Theory and an Application to Prisons," Quarterly Journal of Economics, 112: 1127-1161.

Khalil, F. (1997), "Auditing without Commitment," Rand Journal of Economics, 28: 629-640.

Kofman, F. and J. Lawarrée (1993), "Collusion in Hierarchical Agency", Econometrica, 61: $629-656$. 
Laffont, J.-J. and J. Tirole (1986), "Using Cost Observation to Regulate Firms", Journal of Political Economy, 94: 614-641.

Laffont, J.-J. and J. Tirole (1991), "Privatization and Incentives", Journal of Law, Economics and Organization, 7: 84 - 105.

Laffont, J.-J. and J. Tirole (1992), "Cost Padding, Auditing and Collusion", Annales d'Économie et Statistique, 25-26: 205 - 226.

Megginson, W.L., Nash, R.C. and M. Van Randenborgh (1994), "The Financial and Operating Performance of Newly Privatized Firms: An International Empirical Analysis", Journal of Finance, 49: 403 - 452.

Megginson, W.L. and J.F. Netter (1999), "From State to Market: A Survey of Empirical Studies on Privatization", mimeo, presented at joint conference of SBF Bourse de Paris and New York Stock Exchange, Paris, December 10-11, 1998.

Sappington, D.E. and J.E. Stiglitz (1987), "Privatization, Information and Incentives", Journal of Policy Analysis and Management, 6: 567 - 582.

Schmidt, K.M. (1996a), "The Costs and Benefits of Privatization - An IncompleteContracts Approach", Journal of Law, Economics and Organization, 12: 1 - 24.

Schmidt, K.M. (1996b), "Incomplete Contracts and Privatization", European Economic Review, 40: 569 - 579.

Schmitz, P.W. (2000), "Partial Privatization and Incomplete Contracts: The Proper Scope of Government Reconsidered", mimeo, University of Bonn.

Segal, I. (1998), "Monopoly and Soft Budget Constraint", RAND Journal of Economics, 29: $596-609$.

Vickers, J. and Y.Yarrow (1991), "Economic Perspectives on Privatization", Journal of Economic Perspectives, 5: 111-132. 


\section{Appendix}

Proof of Proposition 1

The proof proceeds in two steps. We first derive and fully characterize the optimal contract for the public and private principal, respectively. Subsequently, we compare managerial effort and the frequency of audits under both regimes.

Step 1: The optimal contract $\left\{\left(p_{j}, \bar{e}_{j}^{a}, \bar{U}_{j}^{a}\right),\left(\underline{e}_{j}^{a}, \underline{U}_{j}^{a}\right)\right\}$ for the principal $j \in\{G, P\}$ if the auditing technology is available therefore minimizes expected costs

$$
\mathcal{C}_{j}=q\left[\underline{\theta}-\underline{e}_{j}+\psi\left(\underline{e}_{j}\right)+\alpha_{j} \underline{U}_{j}\right]+(1-q)\left[\bar{\theta}-\bar{e}_{j}+\psi\left(\bar{e}_{j}\right)+\alpha_{j} \bar{U}_{j}\right]+(1-q) p_{j} c,
$$

where $\alpha_{j}$ is an indicator variable with $\alpha_{P}=1$ and $\alpha_{G}=\frac{\lambda}{1+\lambda}$, subject to the (PC) and (IC) constraints and $p_{j} \in[0,1]$. The Lagrangian of the principal's problem is

$$
\begin{aligned}
L= & q\left[\underline{\theta}-\underline{e}_{j}+\psi\left(\underline{e}_{j}\right)+\alpha_{j} \underline{U}_{j}\right]+(1-q)\left[\bar{\theta}-\bar{e}_{j}+\psi(\bar{e})+\alpha_{j} \bar{U}_{j}\right]+(1-q) p_{j} c \\
& -\lambda_{1}\left\{\bar{U}_{j}-U_{r}\right\}-\lambda_{2}\left\{\underline{U}_{j}-U_{r}\right\} \\
& -\lambda_{3}\left\{\underline{U}_{j}-\bar{U}_{j}-\psi\left(\bar{e}_{j}\right)+\psi\left(\bar{e}_{j}-\Delta \theta\right)+p_{j}(2 \gamma-1) F\right\}-\lambda_{4}\left\{1-p_{j}\right\},
\end{aligned}
$$

with the non-negativity constraints $\bar{e}_{j} \geq 0, \underline{e}_{j} \geq 0$ and $p_{j} \geq 0$ where we have omitted the $(\overline{I C})$ constraint, which will not bind under the optimal contract. The corresponding Kuhn-Tucker conditions are

$$
\begin{aligned}
\frac{\partial L}{\partial \underline{e}_{j}} & =-q\left[1-\psi^{\prime}\left(\underline{e}_{j}\right)\right] \geq 0, \quad \underline{e}_{j} \geq 0 \quad \text { and } \quad \underline{e}_{j} \frac{\partial L}{\partial \underline{e}_{j}}=0 \\
\frac{\partial L}{\partial \underline{U}_{j}} & =q \alpha_{j}-\lambda_{2}-\lambda_{3}=0 \\
\frac{\partial L}{\partial \bar{e}_{j}} & =-(1-q)\left[1-\psi^{\prime}\left(\bar{e}_{j}\right)\right]+\lambda_{3}\left[\psi^{\prime}\left(\bar{e}_{j}\right)-\psi^{\prime}\left(\bar{e}_{j}-\Delta \theta\right)\right] \geq 0, \bar{e}_{j} \geq 0 \quad \text { and } \bar{e}_{j} \frac{\partial L}{\partial \bar{e}_{j}}=0 \\
\frac{\partial L}{\partial \bar{U}_{j}} & =(1-q) \alpha_{j}-\lambda_{1}+\lambda_{3}=0 \\
\frac{\partial L}{\partial p_{j}} & =(1-q) c-\lambda_{3}(2 \gamma-1) F+\lambda_{4} \geq 0, \quad p_{j} \geq 0 \quad \text { and } \quad p_{j} \frac{\partial L}{\partial p_{j}}=0,
\end{aligned}
$$

plus the constraints and their complementary slackness conditions. ¿From $(7)$ and $\psi^{\prime}(0)=0$, we have $\underline{e}_{j}^{a}>0$ and $1=\psi^{\prime}\left(\underline{e}_{j}^{a}\right) \Leftrightarrow \quad \underline{e}_{j}^{a}=e^{*}, j=G, P$. Similarly [see footnote 12], we must also have $\bar{e}_{j}^{a}>0$ so that (9) holds with equality,

$$
1=\psi^{\prime}\left(\bar{e}_{j}^{a}\right)+\frac{\lambda_{3}}{1-q}\left[\psi^{\prime}\left(\bar{e}_{j}^{a}\right)-\psi^{\prime}\left(\bar{e}_{j}^{a}-\Delta \theta\right)\right] .
$$

¿From (8), $\lambda_{2}+\lambda_{3}=\alpha_{j} q$ implies $\lambda_{3} \leq \alpha_{j} q$. Since $\lambda_{4} \geq 0$, (11) yields $p_{j}=0$ if $c(1-q)-$ $\alpha_{j} q(2 \gamma-1) F>0$, or

$$
c>c_{j} \equiv \frac{\alpha_{j} q}{1-q}(2 \gamma-1) F .
$$

Hence, $c>c_{j}$ implies $p_{j}=0$ which together with $(\underline{I C})$ and $(\overline{P C})$ implies $\lambda_{2}=0$ and thus $\lambda_{3}=\alpha_{j} q$. Substituting for $\lambda_{3}$ in (12), the optimal effort if auditing is feasible but the principal 
chooses not to do so is given by

$$
1=\psi^{\prime}\left(\bar{e}_{j}^{a}\right)+\frac{\alpha_{j} q}{1-q}\left[\psi^{\prime}\left(\bar{e}_{j}^{a}\right)-\psi^{\prime}\left(\bar{e}_{j}^{a}-\Delta \theta\right)\right]
$$

and identical to $\bar{e}_{j}^{n a}$ by (5) and (4). Conversely, $c<c_{j}$ contradicts $p_{j}=0$ so that (11) holds with equality. Three cases must be distinguished:

i) for $p_{j}>0$ and $\lambda_{2}=0, \lambda_{3}=\alpha_{j} q>0$ so that we again have $\bar{e}_{j}^{a}=\bar{e}_{j}^{n a}$, i.e., the principal conducts audits only to reduce the manager's informational rent. Observe that for $\lambda_{3}=\alpha_{j} q$ and $c<c_{j}$, (11) yields $\lambda_{4}=\alpha_{j} q(2 \gamma-1) F-(1-q) c>0$. We therefore must have $p_{j}=1$ and the principal audits with probability one. In order for $(\underline{P C})$ to be slack $\left(\lambda_{2}=0\right)$, however, it is necessary that $\psi\left(\bar{e}_{j}^{a}\right)-\psi\left(\bar{e}_{j}^{a}-\Delta \theta\right)-(2 \gamma-1) F \geq 0$ at $\bar{e}_{j}^{a}=\bar{e}_{j}^{n a}$ or

$$
F(2 \gamma-1) \leq \psi\left(\bar{e}_{j}^{n a}\right)-\psi\left(\bar{e}_{j}^{n a}-\Delta \theta\right)=\phi_{j}^{n a} .
$$

Conversely, $F(2 \gamma-1)>\phi_{j}^{n a}$ contradicts $\lambda_{2}=0$. Hence, in this region, $(\underline{P C})$ is binding at the optimum,

$$
p_{j}(2 \gamma-1) F=\psi\left(\bar{e}_{j}^{a}\right)-\psi\left(\bar{e}_{j}^{a}-\Delta \theta\right)=\phi\left(\bar{e}_{j}^{a}\right)
$$

and $\lambda_{2}>0 \Rightarrow \lambda_{3}<\alpha_{j} q$ so that $\bar{e}_{j}^{a}>\bar{e}_{j}^{n a}$ by (5), (4), and (12). There are two possibilities:

ii) if $\lambda_{2}>0$ and $p_{j}<1\left(\lambda_{4}=0\right)$, we have from (11), $\lambda_{3}=\frac{c(1-q)}{(2 \gamma-1) F}$. After substituting for $\lambda_{3}$, $\bar{e}_{j}^{a}$ can be obtained from (12),

$$
1=\psi^{\prime}\left(\bar{e}_{j}^{a}\right)+\frac{c}{(2 \gamma-1) F}\left[\psi^{\prime}\left(\bar{e}_{j}^{a}\right)-\psi^{\prime}\left(\bar{e}_{j}^{a}-\Delta \theta\right)\right] .
$$

Given $\bar{e}_{j}^{a}$, the auditing probability $p_{j}$ is determined by (13),

$$
p_{j}=\frac{1}{(2 \gamma-1) F}\left[\psi\left(\bar{e}_{j}^{a}\right)-\psi\left(\bar{e}_{j}^{a}-\Delta \theta\right)\right] .
$$

iii) for $\lambda_{2}>0$ and $p_{j}=1\left(\lambda_{4}>0\right), \bar{e}_{j}^{a}$ is determined by substituting for $p_{j}=1$ in (13), i.e., $\phi\left(\bar{e}_{j}^{a}\right)=(2 \gamma-1) F$. Note that $\lambda_{4}>0$ requires $\lambda_{3}>\frac{c(1-q)}{(2 \gamma-1) F}$. Using (12), this condition translates into

$$
c<\tilde{c} \equiv \frac{1-\psi^{\prime}\left(\bar{e}_{j}^{a}\right)}{\psi^{\prime}\left(\bar{e}_{j}^{a}\right)-\psi^{\prime}\left(\bar{e}_{j}^{a}-\Delta \theta\right)}(2 \gamma-1) F
$$

Hence, the constraint $p_{j} \leq 1$ is binding and we are in case iii) only if $c$ is sufficiently small. Otherwise, for $c \geq \tilde{c}$, case ii) applies and $p_{j}<1$.

For future reference, also observe that in both cases ii) and iii), $\bar{e}_{j}^{a}$ does not depend on $j$. Furthermore, we have $\lambda_{3} \geq \frac{c(1-q)}{(2 \gamma-1) F}>0$ which together with equation (12) implies that $\bar{e}_{j}^{a} \leq e^{*}$ with $\bar{e}_{j}^{a}=e^{*}$ only for $c=0$ or $F \rightarrow \infty$.

Step 2: We show $p_{P} \geq p_{G}$ and $e_{P}^{a} \leq e_{G}^{a}$ where the latter inequality is strict if and only if $p_{G}=0$ or $(2 \gamma-1) F<\phi_{G}^{n a}$. Since $\bar{e}_{G}^{a}>\bar{e}_{G}^{n a} \Leftrightarrow p_{G}>0 \wedge(2 \gamma-1) F>\phi_{G}^{n a}$ from Step 1, the last claim in Proposition 1 then follows. Again, there are several cases to distinguish: a) for $c>c_{P}$, neither principal monitors and $p_{P}=p_{G}$ follows trivially. Since $\bar{e}_{j}^{a}=\bar{e}_{j}^{n a}$ in this case, we also have $\bar{e}_{P}^{a}<\bar{e}_{G}^{a}$ from Section 2.1. b) for $c_{P} \geq c>c_{G}, p_{P}>p_{G}=0$ and it remains to 
show that $\bar{e}_{P}^{a}<\bar{e}_{G}^{a}$. From Step 1, we know that the effort $\bar{e}_{P}^{a}$ for $p_{P}>0$ is highest in regions ii) and iii) where $\bar{e}_{P}^{a}>\bar{e}_{p}^{n a}$. Hence, $\bar{e}_{P}^{a}$ is determined by [see (12)]

$$
1=\psi^{\prime}\left(\bar{e}_{P}^{a}\right)+\frac{\lambda_{3}}{1-q}\left[\psi^{\prime}\left(\bar{e}_{P}^{a}\right)-\psi^{\prime}\left(\bar{e}_{P}^{a}-\Delta \theta\right)\right] .
$$

with $\lambda_{3} \geq \frac{(1-q) c}{(2 \gamma-1) F}$. Next, as $p_{G}=0, \bar{e}_{G}^{a}=\bar{e}_{G}^{n a}$ and thus is given by

$$
1=\psi^{\prime}\left(\bar{e}_{p}^{a}\right)+\frac{\lambda}{1+\lambda} \frac{q}{1-q}\left[\psi^{\prime}\left(\bar{e}_{G}^{a}\right)-\psi^{\prime}\left(\bar{e}_{G}^{a}-\Delta \theta\right)\right] .
$$

Comparing (*) with (**), we see that $\bar{e}_{P}^{a}<\bar{e}_{G}^{a}$ if only if $\lambda_{3}>\frac{\lambda q}{1+\lambda}$. Now suppose by contradiction that $\lambda_{3} \leq \frac{\lambda q}{1+\lambda}$. Since $\lambda_{3} \geq \frac{(1-q) c}{(2 \gamma-1) F}$ also holds, we must have

$$
\frac{(1-q) c}{(2 \gamma-1) F} \leq \frac{\lambda q}{1+\lambda}
$$

which contradicts $c>c_{G}$. c) Next, consider $c \leq c_{G}$ so that $p_{j}>0, j=G, P$. For $(2 \gamma-1) F \leq$ $\phi_{P}^{n a}$, we are in case i) for both principals. Hence, $p_{P}=p_{G}=1$ and $\bar{e}_{P}^{a}=\bar{e}_{P}^{n a}<\bar{e}_{G}^{n a}=\bar{e}_{G}^{a}$. For $\phi_{G}^{n a} \leq(2 \gamma-1) F$, we know that optimal contracts lie in region ii) or iii) under both ownership structures. From Step 1, $\bar{e}_{j}^{a}$ does not depend on $j$ in this case. We thus have $\bar{e}_{P}^{a}=\bar{e}_{G}^{a}$ and $p_{G}=p_{P}$ by (13). Finally, assume $\phi_{P}^{n a}<(2 \gamma-1) F<\phi_{G}^{n a}$ so that $p_{G}=1$ and $\bar{e}_{G}^{a}=\bar{e}_{G}^{n a}$ for the public principal [region i)] and $\bar{e}_{p}^{a}>\bar{e}_{P}^{n a}$ for the private principal. In order to show that $p_{P}=1$ but $\bar{e}_{P}^{a}<\bar{e}_{G}^{a}$ in this case, one can apply a similar argument as in ii): suppose by contradiction that $p_{P}<1$ so that we are in region ii) for the private principal. In this situation, we must have $c>\tilde{c}$ [otherwise, it would be optimal to set $p_{P}=1$, see (14)] and $\bar{e}_{P}^{a}$ is given by

$$
1=\psi^{\prime}\left(\bar{e}_{P}^{a}\right)+\frac{c}{(2 \gamma-1) F}\left[\psi^{\prime}\left(\bar{e}_{P}^{a}\right)-\psi^{\prime}\left(\bar{e}_{P}^{a}-\Delta \theta\right)\right] .
$$

while $\bar{e}_{G}^{a}$ is determined by (**) so that $c>\tilde{c}$ implies $\bar{e}_{P}^{a}>\bar{e}_{G}^{n a}$. However, since $(\underline{\mathrm{PC}})$ is binding and $(2 \gamma-1) F<\phi_{G}^{n a}$ by assumption, (13) implies

$$
\phi\left(\bar{e}_{P}^{a}\right)=p_{P}(2 \gamma-1) F<p_{P} \phi_{G}^{n a}<\phi_{G}^{n a} \quad \Leftrightarrow \quad \bar{e}_{P}^{a}<\bar{e}_{G}^{n a},
$$

a contradiction. Hence, $p_{P}=1$ which also implies $\bar{e}_{P}^{a}<\bar{e}_{G}^{n a}$.

Proof of Lemma 1: Let

$$
\mathcal{C}_{j}^{a}=q\left\{\underline{\theta}-\underline{e}_{j}^{a}+\psi\left(\underline{e}_{j}^{a}\right)+\alpha_{j} \underline{U}_{j}^{a}\right\}+(1-q)\left\{\bar{\theta}-\bar{e}_{j}^{a}+\psi\left(\bar{e}_{j}^{a}\right)+\alpha_{j} \bar{U}_{j}^{a}\right\}+(1-q) p_{j} c
$$

be the expected operating costs of the firm for the principal $j \in\{G, P\}$ if auditing occurs with positive probability. Similarly, let

$$
\mathcal{C}_{j}^{n a}=q\left\{\underline{\theta}-\underline{e}_{j}^{n a}+\psi\left(\underline{e}_{j}^{n a}\right)+\alpha_{j} \underline{U}_{j}^{n a}\right\}+(1-q)\left\{\bar{\theta}-\bar{e}_{j}^{n a}+\psi\left(\bar{e}_{j}^{n a}\right)+\alpha_{j} \bar{U}_{j}^{n a}\right\}
$$

be the corresponding cost if $p_{j}=0$. We want to show that

$$
\mathcal{C}_{P}^{n a}-\mathcal{C}_{P}^{a}>\mathcal{C}_{G}^{n a}-\mathcal{C}_{G}^{a}
$$


Since $\bar{U}_{j}^{a}=\bar{U}_{j}^{n a}=U_{r}$ and $\underline{e}_{j}^{a}=\underline{e}_{j}^{n a}$, we can confine our attention to differences in $\bar{e}_{j}$ and $\underline{U}_{j}$. Suppose first $(2 \gamma-1) F \leq \phi_{P}^{n a}$. Hence, $\bar{e}_{j}^{a}=\bar{e}_{j}^{n a}, p_{j}=1$ and $\underline{U}_{j}^{a}=\underline{U}_{j}^{n a}-(2 \gamma-1) F \geq U_{r}$ from Proposition 1. Substituting these expressions into $\mathcal{C}_{j}^{n a}-\mathcal{C}_{j}^{a}$ immediately yields

$$
\mathcal{C}_{P}^{n a}-\mathcal{C}_{P}^{a}=q(2 \gamma-1) F-(1-q) c>q \frac{\lambda}{1+\lambda}(2 \gamma-1) F-(1-q) c=\mathcal{C}_{G}^{n a}-\mathcal{C}_{G}^{a} .
$$

Next, consider $\phi_{P}^{n a}<(2 \gamma-1) F \leq \phi_{G}^{n a}$. Again, Proposition 1 implies $\bar{e}_{G}^{a}=\bar{e}_{G}^{n a}, p_{G}=1$, $\bar{e}_{P}^{a}>\bar{e}_{P}^{n a}, \underline{U}_{G}^{a}=\underline{U}_{G}^{n a}-(2 \gamma-1) F \geq U_{r}$ and $\underline{U}_{P}^{a}=U_{r}$. Also recall that $\underline{U}_{P}^{n a}=U_{r}+\phi_{P}^{n a}$ and from the proof of Proposition 1 [Step 2, c)], that $p_{P}=1$ and $e_{P}^{a} \leq e_{G}^{n a}$. Condition (15) simplifies to

$$
q \phi_{P}^{n a}+(1-q)\left\{\bar{e}_{P}^{a}-\bar{e}_{P}^{n a}-\psi\left(\bar{e}_{P}^{a}\right)+\psi\left(\bar{e}_{P}^{n a}\right)\right\}>q \frac{\lambda}{1+\lambda}(2 \gamma-1) F .
$$

Now, (4) implies that $1-\psi^{\prime}(e) \geq \frac{\lambda}{1+\lambda} \frac{q}{1-q}\left[\psi^{\prime}(e)-\psi^{\prime}(e-\Delta \theta)\right]$ for all effort levels $e \leq e_{G}^{n a}$. Hence, $\bar{e}_{P}^{n a}<\bar{e}_{P}^{a} \leq \bar{e}_{G}^{n a}$ implies

$$
\begin{aligned}
(1-q)\left\{\bar{e}_{P}^{a}-\bar{e}_{P}^{n a}-\psi\left(\bar{e}_{P}^{a}\right)+\psi\left(\bar{e}_{P}^{n a}\right)\right\} & =(1-q) \int_{\bar{e}_{P}^{n a}}^{\bar{e}_{P}^{a}}\left[1-\psi^{\prime}(e)\right] d e \\
& \geq(1-q) \int_{\bar{e}_{P}^{n a}}^{\bar{e}_{P}^{a}} \frac{\lambda}{1+\lambda} \frac{q}{1-q}\left[\psi^{\prime}(e)-\psi^{\prime}(e-\Delta \theta)\right] d e \\
& =q \frac{\lambda}{1+\lambda}\left(\phi_{P}^{a}-\phi_{P}^{n a}\right) .
\end{aligned}
$$

Using this inequality and the fact that $\underline{U}_{P}^{a}=U_{r} \Leftrightarrow \phi_{P}^{a}=(2 \gamma-1) F$ (since $\left.p_{P}=1\right)$ yields

$$
\begin{aligned}
q \phi_{P}^{n a}+(1-q)\left\{\bar{e}_{P}^{a}-\bar{e}_{P}^{n a}-\psi\left(\bar{e}_{P}^{a}\right)+\psi\left(\bar{e}_{P}^{n a}\right)\right\} & \geq q \phi_{P}^{n a}+q \frac{\lambda}{1+\lambda}(2 \gamma-1) F-q \frac{\lambda}{1+\lambda} \phi_{P}^{n a} \\
& =q\left(1-\frac{\lambda}{1+\lambda}\right) \phi_{P}^{n a}+q \frac{\lambda}{1+\lambda}(2 \gamma-1) F \\
& >q \frac{\lambda}{1+\lambda}(2 \gamma-1) F
\end{aligned}
$$

for $\phi_{P}^{n a}>0$ and $\lambda<<\infty$ as required. Finally, consider $\phi_{G}^{n a}<(2 \gamma-1) F$ which implies $p_{G}=p_{P}$, $\bar{e}_{P}^{a}=\bar{e}_{G}^{a}>\bar{e}_{G}^{n a}$ and $\underline{U}_{j}^{a}=U_{r}$ by Proposition 1. Condition (15) can be written as

$q \phi_{P}^{n a}+(1-q)\left\{\bar{e}_{P}^{a}-\bar{e}_{P}^{n a}-\psi\left(\bar{e}_{P}^{a}\right)+\psi\left(\bar{e}_{P}^{n a}\right)\right\}>q \frac{\lambda}{1+\lambda} \phi_{G}^{n a}+(1-q)\left\{\bar{e}_{G}^{a}-\bar{e}_{G}^{n a}-\psi\left(\bar{e}_{G}^{a}\right)+\psi\left(\bar{e}_{G}^{n a}\right)\right\}$,

which is for $\bar{e}_{P}^{a}=\bar{e}_{G}^{a}$ equivalent to

$$
q \phi_{P}^{n a}+(1-q)\left\{\bar{e}_{G}^{n a}-\bar{e}_{P}^{n a}-\psi\left(\bar{e}_{G}^{n a}\right)+\psi\left(\bar{e}_{P}^{n a}\right)\right\}>q \frac{\lambda}{1+\lambda} \phi_{G}^{n a} .
$$

Since $\bar{e}_{P}^{n a}<\bar{e}_{G}^{n a}$, applying the same argument as above yields

$$
(1-q)\left\{\bar{e}_{G}^{n a}-\bar{e}_{P}^{n a}-\psi\left(\bar{e}_{G}^{n a}\right)+\psi\left(\bar{e}_{P}^{n a}\right)\right\}=\int_{\bar{e}_{P}^{n a}}^{\bar{e}_{G}^{n a}}\left[1-\psi^{\prime}(e)\right] d e>q \frac{\lambda}{1+\lambda}\left(\phi_{G}^{n a}-\phi_{P}^{n a}\right),
$$

and the result follows. 